\title{
Noisy Filtered Sparse Processes: Reconstruction and Compression
}

\author{
Manqi Zhao and Venkatesh Saligrama \\ Department of Electrical and Computer Engineering \\ Boston University, MA 02215 \\ \{srv, mqzhao\}@bu.edu
}

\begin{abstract}
In this paper we consider estimation and compression of filtered sparse processes. Specifically, the filtered sparse process is a signal $x \in \mathbb{R}^{n}$ obtained by driving a k-sparse signal $u \in \mathbb{R}^{n}$ through an arbitrary unknown stable discretelinear time invariant system $H$ of a known order. The signal $x(t)$ is measured noisily. We consider estimation of $x(t)$ from noisy measurements. We also consider compression of $x(t)$ by means of random projections analogous to compressed sensing. For different cases including AR and MA systems we show that $x$ can indeed be reconstructed from $O(k \log (n))$ measurements. We develop a novel LP optimization algorithm and show that both the unknown filter $H$ and the sparse input $u$ can be reliably estimated.
\end{abstract}

\section{INTRODUCTION}

In this paper we develop methods for estimation and compression of filtered sparse processes. These types of processes naturally arise in reflection seismology [1], neuronal spike trains [2] and communications [3], [4].

A sparse input $u(t)$ is filtered by an unknown infinite impulse response (IIR) discrete time stable linear filter $H$ and the resulting output

$$
x(t)=(H u)(t)=\sum_{i} u\left(\tau_{i}\right) h\left(t-\tau_{i}\right)
$$

is measured in Gaussian noise, namely, $y(t)=x(t)+w(t)$ for $t=0,1, \ldots, n-1$. The goal is to detect $u(t)$, and estimate the filter $H$. The main approach heretofore proposed for blind de-convolution involves heuristic iterative block decomposition schemes (first proposed in [5]). Here the filter and sparse inputs are alternatively estimated by holding one of them constant. While these algorithms can work in some cases, no systematic performance guarantees currently exist. We explore a convex optimization framework for blind de-convolution.

In addition we consider the compressed sensing problem, namely, $x(t)$ is compressed by means of a random Gaussian filter ensemble, as described in Figure 1 and the resulting output is measured noisily. Analogously, we can consider a random excitation model as in Figure 2. Our goal is to characterize the minimum number of random samples required for accurate identification of the system.

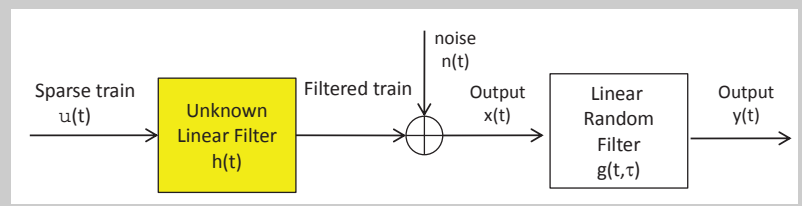

Fig. 1. Compressed Blind de-convolution.

This research was supported by NSF CAREER award ECS 0449194 and NSF Grant 0932114

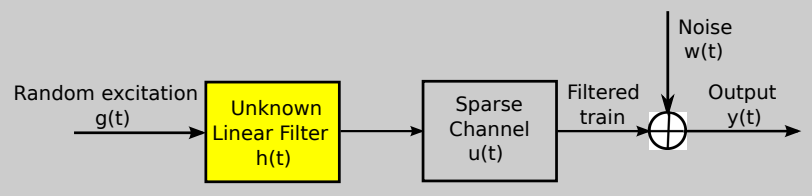

Fig. 2. Random Excitation Model: SYSID

\section{A. Comparison with Compressed Sensing}

Note that this is significantly different from the standard Compressed sensing(CS) [6] problem. In standard CS we have a signal or image, $x \in \mathbb{R}^{n}$, which is sparse in some transform domain. Specifically, there is a known orthonormal matrix $H$ such that the transformed signal $u=H^{T} x$ is k-sparse, namely, has fewer than $k$ non-zero components. A matrix $G \in \mathbb{R}^{m \times n}$ then maps $x$ to measurements $y=G x=G H u$. For suitable choices of matrices $G$, such as those satisfying the so called Restricted Isometry Property (RIP), the k-sparse signal $u$ can be recovered with $O(k \log (n / k))$ measurements as a solution to a convex optimization problem:

$$
\min \|u\|_{1} \text { subject to } y=G H u
$$

This result holds for all sparsity levels $k \leq \alpha n, \alpha<1$, for sufficiently small $\alpha$. There has been significant effort in CS in recent years leading to various generalizations of this fundamental result including important extensions to approximately sparse $x$ and noisy measurements.

This paper is a significant extension of CS to cases where $H$ is not only non-orthonormal but also unknown. Specifically, $H$, is a causal discrete linear time invariant system (LTI) with an unknown impulse response function $h(\cdot)$ as described above. Consequently, a typical signal $x(\cdot)$ is neither sparse nor approximately sparse.

\section{B. Our Approach}

Our goal is to determine the conditions under which $x$ can be recovered uniquely from samples $y=G x=G H u$ using a convex optimization algorithm. The main difficulty is that $x$ need not be sparse and $G H$ may not satisfy RIP even when $H$ is known but not orthonormal.

Consider a one-dimensional piecewise constant signal, $x$, is not sparse but does have a sparse derivative, namely, $u(t)=$ $x(t)-x(t-1)$ is sparse. Clearly, the signal $x$ can represented as an output of an (integral) operator $H$ acting on a sparse input $u$, namely, $x=H u$. However, $H$ is no longer orthonormal. Suppose we now filter $x$ through an LTI system $G$ whose impulse response is $g(t)$. Mathematically, we have,

$$
y(t)=(g * x)(t)=(g * h * u)(t)=(h * g * u)(t)
$$


Since the composite system $g * h$ is LTI we have that,

$$
\tilde{y}(t) \triangleq y(t)-y(t-1)=g *(x(t)-x(t-1))=(g * u)(t)
$$

Now we are in the familiar situation of $\tilde{y}=G u$ of the standard CS problem, except that $G$ is a Toeplitz matrix. Consequently, if the Toeplitz matrix $G$ satisfies the RIP property (see [4], [7] and references therein) we can recover $\tilde{y}$ using standard tools in CS. Note that this idea generalizes to arbitrary but known finite dimensional stable LTI systems, $H$. However, the question arises as to how to deal with unknown system $H$. For $\mathrm{AR}$ processes it is possible to obtain a linear characterization and one can jointly solve for both the AR parameters and the sparse input.

The paper is organized as follows. The mathematical formulation of the problem is presented in Section II. Section III presents solutions for AR filtered processes. Section IIIA addresses the blind-deconvolution problem, which can be regarded as a noisy version of our problem. In Section IV we develop reconstruction and compression algorithms for MA processes. Section V describes extensions to ARMA process. Simulation results are presented in Section VI.

\section{COMPRESSION AND RECONSTRUCTION}

Our objective is to reconstruct the process $x(t)$ from a number of linear and non-adaptive measurements. Note that in standard CS setup (see Equation 1), the signal $x$ is assumed to be sparse in some known transform space. In our problem the system, $H$, is unknown and the main contribution of this paper is to solve this new problem efficiently for some special cases. We consider two types of compressed sensing scenarios:

\section{A. Toeplitz Matrices}

Here we obtain $m$ measurements by applying the sensing matrix $G$ to signal $x=\left[x_{0}, \cdots, x_{n-1}\right]^{T}$ to obtain

$$
y=G x
$$

where

$$
G=\left[\begin{array}{ccccccc}
g_{n-m} & g_{n-m-1} & \cdots & g_{0} & \cdots & 0 & 0 \\
g_{n-m+1} & g_{n-m} & \cdots & g_{1} & g_{0} & \cdots & 0 \\
\vdots & \vdots & \ddots & \vdots & \ddots & \ddots & 0 \\
g_{n-1} & g_{n-2} & \cdots & \cdots & \cdots & g_{1} & g_{0}
\end{array}\right]
$$

where $g_{i}$ 's are IID Gaussian distributed $\mathcal{N}(0,1)$ or equiprobable IID Bernoulli \pm 1 . Here the Toeplitz matrix $G$ preserves the shift-structure of the signal. Roughly speaking, assume $u^{\prime}$ is a time-shifted version of $u$ (disregarding the boundary effect), then $G u^{\prime}$ is also just a time-shifted version of $G u$. This is particularly suitable for the random excitation model of Figure 2.

For notational purposes $x^{[s]}$ (or $G^{[s]}$ ) is used to denote the sub-vector of $x$ (or submatrix of $G$ ) that is composed of the last $s$ components (or $s$ rows) of $x$ (or $G$ ). Denote

$$
Y \triangleq\left[\begin{array}{cccc}
y_{p-1} & \cdots & y_{1} & y_{0} \\
y_{p} & \cdots & y_{2} & y_{1} \\
\vdots & \ddots & \vdots & \vdots \\
y_{m-2} & \cdots & y_{m-p} & y_{m-p-1}
\end{array}\right]
$$

\section{B. Random Projections}

Here we consider randomly projecting the raw measurements $x(t)$, namely,

$$
y(t)=\sum_{\tau=0}^{n-1} g_{t, \tau} x(\tau), t=0,1, \ldots, m
$$

where, $g_{t, \tau}$ 's are IID Gaussian distributed $\mathcal{N}(0,1)$ or equiprobable IID Bernoulli \pm 1 . We call this time-variant system as random projections because in its matrix form $Y=$ $G X$, the sensing matrix $G$ is a random matrix without any Toeplitz structure. The reason for choosing random projections over random filters is that IID random Gaussian/Bernoulli matrix ensembles have superior RIP constants. The optimal RIP constants for Toeplitz constructions has not been fully answered. Nevertheless, note that to form the matrix $Y$ with random projections requires significantly more projections. This is because we can no longer exploit the shift-invariant property of convolutions. For a $p$ th order model random projections require $p$ times more measurements in order to form the matrix $Y$. Our goal is to reconstruct $x, u$, and $H$ from both uncompressed and compressed measurements that are possibly noisy.

\section{III. $\ell_{1}$-MINIMIZATION Algorithm FOR AR Models}

An autoregressive model is known as an "all-pole" model, and has the general form

$$
x(t)+\sum_{i=1}^{p} a_{i} x(t-i)=u(t)
$$

where $u(t)$ is a sparse driving process. We assume the vector $u=\left[u_{0}, \cdots, u_{n-1}\right]^{T}$ is $k$-sparse, that is, there are at most $k$ non-zero components in $u$. The task in this section is to find the AR model coefficients $a=\left[a_{1}, \cdots, a_{p}\right]^{T}$ and the driving process $u=\left[u_{0}, \cdots, u_{n-1}\right]^{T}$ from the measurement $y$. In this paper, we assume that the AR model is stable, that is, the magnitude of all the poles of the system is strictly smaller than 1. In later discussion, we use $x_{t}$ or $x(t)$ interchangeably for convenience of exposition.

Combining Equation 2 and 5 and exploiting the shiftproperty of $G$, we have the following equation.

$$
Y a+y^{[m-p]}=G^{[m-p]} u
$$

where $a=\left[a_{1}, \cdots, a_{p}\right]^{T} \in \mathbb{R}^{p}$ and $u \in \mathbb{R}^{n}$ ( $k$-sparse) need to be decoded from the model.

Since the AR model is unknown, standard CS decoding algorithms (e.g., Basis Pursuit [8]) can not be directly applied to this problem. However, we can regard the signal $(u, a)$ (the original signal $u$ together with the unknown coefficients $a$ ) as the new input to the model and $(u, a)$ is still sparse if $p$ (the length of $a$ ) is small. It may appear that the problem can be reduced to the standard CS problem, namely,

$$
y^{[m-p]}=\left[\begin{array}{ll}
G^{[m-p]} & Y
\end{array}\right]\left(\begin{array}{c}
u \\
a
\end{array}\right)
$$

Notice however that the composite matrix, $\left[G^{[m-p]} Y\right]$, does not necessarily satisfy RIP property. With this in mind we solve the following $\ell_{1}$ minimization algorithm

$$
\min _{u \in \mathbb{R}^{n}, a \in \mathbb{R}^{p}}\|u\|_{1} \quad \text { s.t. } \quad Y a+y^{[m-p]}=G^{[m-p]} u
$$


More generally, for the noisy sensing model $y=G x+w$ where $w$ is Gaussian noise, the above LP algorithm is relaxed to LASSO,

$$
\min _{u \in \mathbb{R}^{n}, a \in \mathbb{R}^{p}} \frac{1}{2}\left\|Y a+y^{[m-p]}-G^{[m-p]} u\right\|_{2}^{2}+\lambda\|u\|_{1}
$$

where $\lambda$ is a tuning parameter that adapts to the noise level.

Before stating the main result, we recall that for every integer $S$ the restricted isometry constant [9], $\delta_{S}$ is defined to be the smallest quantity such that $G_{T}^{[m-p]}$ obeys

$$
\left(1-\delta_{S}\right)\|x\|_{2}^{2} \leq\left\|G_{T}^{[m-p]} x\right\|_{2}^{2} \leq\left(1+\delta_{S}\right)\|x\|_{2}^{2}
$$

for all subsets $T \subset\{0,1, \cdots, n-1\}$ of cardinality at most $S$ and all $\left(x_{j}\right)_{j \in T}$. Here $G_{T}$ denotes the submatrix of $G$ with column indices in $T$.

We need the following assumptions before we state our theorem.

(1) Constant Order: We assume that $p$, the order of AR process $x(n)$, is a constant (i.e., $p$ does not scale with $n, m, S$ ). (2) Exponential Decay: Suppose the impulse response $|h(i)|$ of the AR model satisfies $|h(i)| \leq M \rho^{i}$ for some constant $M$ and $0<\rho<1$.

(3) Spike Amplitude: We assume that any spike is bounded, $\beta_{\min } \leq\left|u_{k}\right| \leq \beta_{\max }, \forall k \in \operatorname{Supp}\left(u^{*}\right)$, where $u^{*}$ is the true spike train.

(4) Distance between Spikes: We define the constant $l$ := $\left(\log \left(\frac{2}{1-\rho}\right)+p \log \left(\frac{6 \beta_{\max } M}{\beta_{\min }}\right)\right) / \log \left(\rho^{-1}\right)+p$ and impose the condition that any two spikes, $u_{i}^{*}, u_{j}^{*}$ satisfy $|i-j|>$ $l, i \neq j$. This implies that the sparsity $k:=\left|\operatorname{Supp}\left(u^{*}\right)\right| \leq$ $\min \{S / l, S / 3\}$.

Theorem 1. Suppose assumptions 1-4 above are satisfied. Let the integer $S$ satisfy $\frac{\delta_{S}}{1-3 \delta_{S}}<1$. If $u^{*}(\cdot)$ is the true driving process in Equation 5 then it is the unique minimizer of

$$
\min _{u \in \mathbb{R}^{n}, a \in \mathbb{R}^{p}}\|u\|_{1} \quad \text { s.t. } \quad Y a+y^{[m-p]}=G^{[m-p]} u
$$

The detailed proof of this theorem can be found in [10]. Here we just provide some intuitions of the proof. The proof is based on duality. Duality theory says that $u^{*}$ is the unique minimizer of the primal problem if there exists a dual vector $\pi$ with the following properties:

$$
\begin{aligned}
& \text { 1) }\left(\pi^{T} G^{[m-p]}\right)_{i}=\operatorname{sgn}\left(u_{i}^{*}\right) \text { for all } i \in \operatorname{Supp}\left(u^{*}\right) \text {, } \\
& \text { 2) }\left|\left(\pi^{T} G^{[m-p]}\right)_{j}\right|<1 \text { for all } j \notin \operatorname{Supp}\left(u^{*}\right), \\
& \text { 3) } \pi^{T} Y=0 .
\end{aligned}
$$

where $\operatorname{sgn}\left(u_{i}^{*}\right)$ denotes the sign of $u_{i}^{*}\left(\operatorname{sgn}\left(u_{i}^{*}\right)=0\right.$ for $u_{i}^{*}=$ $0)$ and $\operatorname{Supp}\left(u^{*}\right)$ denotes the support of vector $u^{*}$. The above set of conditions is an application of the Karush-Kuhn-Tucker (KKT) condition and we call it as the Dual Optimal Condition (DOC). The main idea of our proof is to construct a $\pi$ that satisfies the DOC.

The assumptions in the theorem require that the driving process $u(n)$ is sparse enough and any two spikes $\left(u_{i}, u_{j}\right)$ are reasonably separated. This assumption is essential. To see this, consider the following example: Consider a 1st order AR process with only two entries of $u(n)$ non-zero. Specifically, we choose $u_{0}^{*}=u_{1}^{*}=1$ and $u_{i}^{*}=0(\forall i>1)$. Here the two spikes are next to each other and this violates the last assumption $\left|i-i^{\prime}\right|>l, \forall i, i^{\prime} \in \operatorname{Supp}\left(u^{*}\right)$ in Theorem 1 .
Under this setup $\left[x_{0}, x_{1}, \cdots, x_{n-1}\right]^{T}=[1,1+\alpha, \alpha(1+$ $\left.\alpha), \cdots, \alpha^{n-2}(1+\alpha)\right]^{T}$ where we pick the pole $\alpha=-1 / 2$. We choose $\pi$ to satisfy condition 1 and 2, then we can easily bound $\pi^{T} Y \geq 1-\sum_{j=2}^{n-1}|\alpha|^{j-2}(1-|\alpha|)>0$, which implies that condition 3 in DOC does not hold. Hence there does not exist a $\pi$ that satisfies DOC.

Remark III.1. Note that by using random convolutions we can naturally exploit shift-invariance property. Since $Y \in$ $\mathbb{R}^{(m-p) \times p}$ as in Equation 3 is a partial Toeplitz matrix, we only need $m$ output measurements. In contrast for a random projection, since we can no longer exploit this property, we would require $O(m p)$ measurements.

Theorem 1 can be generalized to an ARMA model when the zero locations are known. We will state this when we discuss iterative schemes for estimating ARMA models in Section V.

\section{A. Noisy Blind-deconvolution}

We consider the noisy blind-deconvolution problem with IID Gaussian noise, $w_{i} \sim \mathcal{N}\left(0, \sigma^{2}\right)$, and measurements

$$
y(t)=x(t)+w(t)
$$

where $x(t)$ is modeled by the AR process $x(t)+\sum_{i=1}^{p} a_{i} x(t-$ $i)=u(t)$. This problem is called Blind deconvolution [11]. To the best of our knowledge, in the noisy setup even this simplified problem ( $G$ to be identity) is still not solved in literature. Therefore, we focus on the uncompressed noisy version here. The noisy compressed version is technically more involved and will be reported elsewhere.

Replacing $x(t)$ with $y(t)-w(t)$ in the AR model,

$$
y(t)+\sum_{i=1}^{p} a_{i} y(t-i)=u(t)+e(t)
$$

where we denote $e(t):=w(t)+\sum_{i=1}^{p} a_{i} w(t-i)$.

Again by introducing matrix $Y$ (Equation 3 ) we have the matrix-form system model

$$
y+Y a=u+e
$$

For this noisy model, LASSO is applied:

$$
\min _{u \in \mathbb{R}^{n}, a \in \mathbb{R}^{p}} \frac{1}{2}\|y+Y a-u\|_{2}^{2}+\lambda\|u\|_{1}
$$

We will show in the following that the solution of LASSO is just a small perturbation from the true $a^{*}$ and $u^{*}$. We also introduce some notation and technical conditions first. Denote

$$
X:=\left[\begin{array}{ccc}
0 & \cdots & 0 \\
x_{0} & \cdots & 0 \\
\vdots & \ddots & \vdots \\
x_{p-1} & \cdots & x_{0} \\
\vdots & \vdots & \vdots \\
x_{n-2} & \cdots & x_{n-p}
\end{array}\right]
$$

Denote the support of $u^{*}$ as $I$. We define $X_{1}$ as the matrix comprising of the rows of $X$ indexed by $I$ and $X_{2}$ as the matrix comprising of the rows of $X$ indexed by $I^{c}$. We also denote $x_{\max }=\max _{i}\left|x_{i}\right|, u_{\min }=\min _{i \in I}\left|u_{i}\right|$ and $a_{\max }=$ $\max _{i}\left|a_{i}\right|$.

We assume that the AR process $x(n)$ satisfies the following set of conditions. 


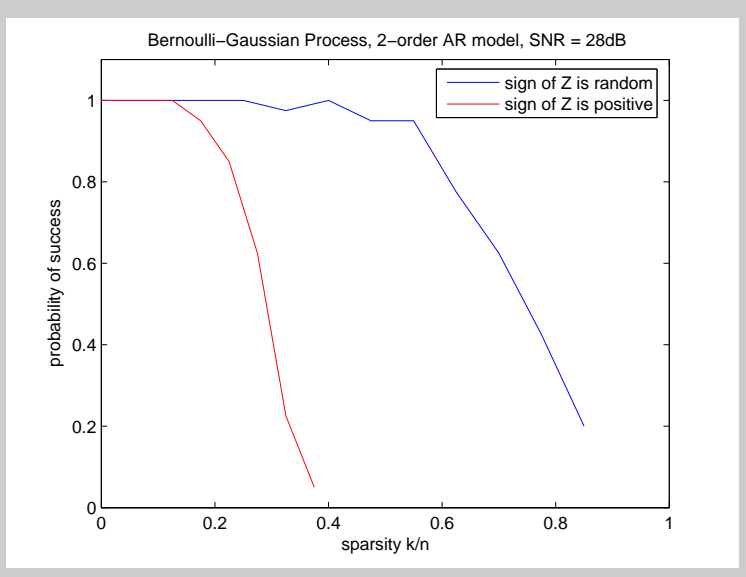

Fig. 3. Comparison of two sign conditions for $u$. The AR model is $x_{t}-1.4 x_{t-1}+0.45 x_{t-2}=u_{t}$. Noises ere added to the measurements and $S N R=28 \mathrm{~dB}$. The blue curve corresponds to the scenario when $\operatorname{sgn}\left(u_{i}\right)\left(u_{i}\right.$ is a spike) is Bernoulli \pm 1 . The red curve corresponds to the case when the sign of any spike $u_{i}$ is always +1 . Each point on the curve is an average over 40 trials. In the first scenario (blue curve) much more spikes can be added given the same level of success probability.

(1) The smallest eigenvalue $\lambda_{\min }\left(X_{2}^{T} X_{2}\right) \geq \frac{\|x\|_{2}^{2}}{c} \geq$ $\frac{4 n p \sigma^{2}}{(\sqrt{2}-1)^{2}}$ for some constant $c>1$.

(2) $\left\|X_{1}^{T} \operatorname{sgn}\left(z_{I}^{*}\right)\right\|_{\infty} \leq\|x\|_{2} \sqrt{\log n}$,

(3) $x_{\max } \geq 2 \sigma \sqrt{\log n}$ and $\frac{x_{\max }^{2}}{\|x\|_{2}^{2}} \leq$ $\min \left\{\frac{1}{4 c \sqrt{2 p n}},\left(\frac{1}{24 c p \sqrt{\log n}}\right)^{2}\right\}$.

In practice, condition (1) is generally satisfied. For instance, if the signal $x$ is persistent, $\frac{1}{\|x\|_{2}} X_{2}^{T} X_{2}$ converges to a constant invertible matrix. Condition (3) is also standard in compressed sensing, which roughly says $S N R \geq O(\log n)$ is required. In addition, we also need the assumption that no components are dominantly large (compared with the total energy of $x$ ). The upper bound for $x_{\max } /\|x\|_{2}$ can be relaxed but the current setup simplifies the analysis.

Condition (2) is new. We present two contrasting examples to justify it. In the first example, each spike in $u_{I}$ is positive or negative with equal probability (i.e. $\operatorname{sgn}\left(u_{I}\right)$ is Bernoulli \pm 1 ). In this case, $X_{1}^{T} \operatorname{sgn}\left(u_{I}^{*}\right)$ behaves like a sub-Gaussian sum and standard analysis can show it is upper bounded by $\|x\|_{2} \sqrt{\log n}$ with high probability. In the second example, all the spikes in $u_{I}$ are of the same sign, say positive. In this case each entry in $X_{1}^{T}$ and $\operatorname{sgn}\left(u_{I}^{*}\right)$ is positive and the inner product of these two aligned signals is much larger than the first example. This phenomena is also illustrated in the experiments shown in Figure 3.

Theorem 2. Denote $P:=I-Y\left(Y^{T} Y\right)^{-1} Y^{T}$ and assume condition (1),(2) and (3) stated above are satisfied. We also assume parameter $\lambda$ is chosen such that $\lambda \geq 6 \sigma p a_{\max } \sqrt{\log n}$ and $u_{\min } \geq 2 \lambda$, the solution to LASSO (14) is given by

$$
\begin{aligned}
\hat{u}_{I} & =\left(P_{I}^{T} P_{I}\right)^{-1}\left(P_{I}^{T} e-\lambda \operatorname{sgn}\left(u_{I}^{*}\right)\right)+u_{I}^{*} \\
\hat{u}_{I^{c}} & =0 \\
\hat{a} & =-\left(Y^{T} Y\right)^{-1} Y^{T}(y-\hat{u})
\end{aligned}
$$

and we have $\operatorname{sgn}\left(u^{*}\right)=\operatorname{sgn}(\hat{u})$ with probability at least $1-$
$8 p / n-(p+1) 2^{-n / 5}$.

Remark: The assumption $u_{\text {min }} \geq 2 \lambda$ implicitly implies an SNR bound $O(\log n)$ for the smallest spike. The assumption $\lambda \geq 6 \sigma p a_{\max } \sqrt{\log n}$ ensures $\lambda$ to be sufficiently large so that every non-spike element is shrunk to zero by the Lasso estimator. It is hard to analyze the case when parameter $\lambda$ is smaller because in this case it is not clear how to construct $\hat{u}_{I^{c}}$ which is critical for tractable KKT analysis. The choice of $\hat{u}$ in the Theorem 2 is motivated by the proof techniques used in [12] and the main idea of of proof is to show that $\hat{u}$ given by the theorem satisfies KKT conditions. And this can be achieved via concentration of measure argument. The detailed proof of Theorem 2 is presented in [10].

\section{Moving Average Model}

Here we consider blind deconvolution problems that involve moving average models. Specifically, let $H$ be an finite impulse response system of order $p$. We denote by $h(k)$ the kth coefficient in the impulse response. Suppose $u(\cdot)$ is a sparse signal, which is filtered through $H$, namely,

$$
x(t)=\sum_{\tau=0}^{p} h(\tau) u(t-\tau), t=0,1, \ldots, n
$$

In the associated compressed sensing MA problem, we pass the filtered sparse signal through another random filter $g(\cdot)$.

$$
y(t)=\sum_{q=0}^{t} g(q) x(t-q)+w(t), t=n-m, \ldots, n
$$

where $w(t)$ is assumed to be IID Gaussian noise with mean zero and variance $\sigma_{w}^{2}$. Note that we collect the last $m$ sequential measurements out of the $n$ possible measurements.

We assume that both the system $H$ and the input signal $u$ is unknown. However, we impose additional assumptions on $u$ and the FIR system $H$. Specifically, we assume that the FIR system $H$ is minimum phase. The input $u$ is assumed to be a Bernoulli-Gaussian process. the components of the signal are i.i.d. obeying the mixture distribution:

$$
u_{i} \stackrel{d}{\sim} P_{u}=\alpha \mathcal{N}\left(\mu_{1}, \sigma_{1}^{2}\right)+(1-\alpha) \mathcal{N}\left(\mu_{0}, \sigma_{0}^{2}\right)
$$

i.e., the components $\left\{u_{i}\right\}$ are i.i.d. $P_{u}$ defined above. It is easy to see that for $\mu_{1}=1, \mu_{0}=0$ for $\sigma_{0}=0$ this mixture model for large enough $n$ results in an approximately $k=\alpha n$ sparse sequence. We use $\sigma_{1}=0$ to model a binary discrete case and $\sigma_{1}=1$ to model a continuous valued case.

Since convolutions are commutative we can rewrite the model as follows:

$$
y(t)=\sum_{\tau=0}^{p} h(\tau) z(t-\tau)+w(t), t=n-m, \ldots, n
$$

where, $z(\cdot)$ is given by, $z(t)=\sum_{\tau=0}^{t} g(\tau) u(t-\tau)$.

Our goal now is to estimate the FIR filter $H$ and the sparse signal $u$ as follows:

(1) Estimate $H$ through spectral factorization.

(2) Estimate $u$ using LASSO.

To this end let $r_{z}^{n}(\tau)$ denote the empirical auto-correlation of a random signal, $z$, at delay $\tau$, namely,

$$
r_{z}^{n}(\tau)=\frac{1}{n-\tau} \sum_{t=0}^{n-\tau} z(t) z(t+\tau)
$$


Let $r_{z}(\tau)=\lim _{t \rightarrow \infty} E(z(t) z(t+\tau))$. We estimate the autocorrelation coefficients as follows:

$$
r_{h}^{n}(0)=\frac{r_{y}^{n}(0)-\sigma_{w}^{2}}{r_{u}(0)}, r_{h}^{n}(\tau)=\sum_{t=0}^{p-\tau} h(t) h(t+\tau)=\frac{r_{y}^{n}(\tau)}{r_{u}(0)}
$$

Our next step is to ensure that the fourier transform of the autocorrelation coefficients is positive on the unit disc. If a system $H$ is minimum phase, i.e., does not contain zeros either on or outside the unit disc, the fourier transform of the auto-correlation coefficients is positive, namely, the power spectral density is positive. However, this is not necessarily true for estimated auto-correlation coefficients. Nevertheless, we can correct the autocorrelation coefficient estimates by means of the following Semi-Definite Program (SDP). The SDP formulation is well-known and we refer the reader to [13] for further details.

$$
\min \left\|\hat{r}_{h}-r_{h}^{n}\right\|_{2}
$$

subject to,

$$
\left[\begin{array}{cc}
P & R_{h} \\
R_{h}^{T} & \hat{r}_{h}(0)
\end{array}\right]-\left[\begin{array}{cc}
0 & 0 \\
0 & P
\end{array}\right] \geq 0
$$

where,

$$
R_{h}=\left[\begin{array}{llll}
\hat{r}_{h}(p) & \hat{r}_{h}(p-1) & \ldots & \hat{r}_{h}(1)
\end{array}\right]^{T}
$$

and $P \in \mathbb{R}^{(p-1) \times(p-1)}$ is any symmetric matrix.

The solution to the above optimization problem has two important properties:

(1) The Fourier transform of the corrected autocorrelation coefficients $\hat{r}_{h}(\cdot)$ is guaranteed to be positive on the unit disc. (2) The solution $\hat{r}_{h}(\cdot)$ is the closest to the estimated spectrum $r_{h}^{n}(\cdot)$ that has this positivity property.

Next we estimate the positive spectral factors, $\hat{H}$, using any one of the well-known spectral factorization methods.

Now we are ready to estimate the sparse input $u$. To do this we solve a LASSO optimization problem. Specifically, we have for the uncompressed case,

$$
\min _{u}\left\|x(t)-\sum_{\tau=0}^{p} \hat{h}(\tau) u(t-\tau)\right\|_{2}^{2}+\|u\|_{1}
$$

and similarly for the compressed case,

$$
\min _{u}\left\|y(t)-\sum_{q=0}^{t} g(q) \sum_{\tau=0}^{p} \hat{h}(\tau) u(t-q-\tau)\right\|_{2}^{2}+\|u\|_{1}
$$

We have the following theorem in this context.

Theorem 3. Consider the setup of the moving average process along with the algorithm (spectral factorization followed by LASSO) described above. Then if the number of measurements satisfies, $m=\Omega(k \log (n / k))$, the solution $u^{*}$ to LASSO recovers the true support with high probability.

We present a brief outline of the proof here. The proof is based on ensuring that the autocorrelation estimates are accurate with high probability. This requires that the positive definite estimates derived from the SDP algorithm above are sufficiently close to the true autocorrelation estimates. Second we need to make sure that the positive spectral factors are sufficiently close to the true positive spectral factors. This is ensured by direct application of results of Boche [14]. Finally, we establish that LASSO is robust to the inaccurate estimation of the parameters.

\section{ARMA MODEL}

In this section, we consider autoregressive moving average (ARMA) models, i.e., the process contains both poles and zeros in the transform function. The ARMA model takes the form

$$
x(t)+\sum_{i=1}^{p} a_{i} x(t-i)=u(t)+\sum_{i=1}^{q} b_{i} u(t-i)
$$

Again we use random convolution (Equation 2) to obtain the measurement $y=G x$ where $G$ is a Toeplitz matrix as defined in Section II.

Similar to the simplification we have done in Section II, we randomly project the output $x(t)$ using a random filter $g(\cdot)$ or by employing random projections as in Equations 2, 4. For the randomly filtered setting, this results in the following equation:

$$
Y a+y^{[m-p]}=G^{[m-p]} B u
$$

where, $B$ is the toeplitz matrix formed from $b_{i}$.

When the coefficients $b$ are known, this setting is close to the AR setup for which we derived the solution in Theorem 1. It turns out that this solution generalizes to this setting where the coefficients $b$ are known, i.e.,

Theorem 4 (Known Zero Locations). Given the same technical conditions as Theorem 1 and assume $u^{*}$ is the original sparse spike train that generates the ARMA process. Then $u^{*}$ is the unique minimizer of

$$
\min _{u \in \mathbb{R}^{n}, a \in \mathbb{R}^{p}}\|u\|_{1} \text { s.t. } \quad Y a+y^{[m-p]}=G^{[m-p]} B u
$$

We are now left to estimate the parameters $b, a, u$ for the general situation where neither the ARMA parameters nor the input is known. Our algorithm is iterative, namely, we fix $b$ and estimate $u, a$. Then fixing $u$ and $a$ we then estimate $b$. Estimation of $b$ reduces to a standard least-squares regression problem. For estimating $u, a$ we solve the following LASSO based on Theorem 4:

$$
\min _{u \in \mathbb{R}^{n}, a \in \mathbb{R}^{p}}\|u\|_{1} \quad \text { s.t. } \quad\left\|Y a+y^{[m-p]}-G^{[m-p]} B u\right\|_{2} \leq \epsilon
$$

Here $\epsilon>0$ is required, even though there may not be any noise, to compensate for uncertainty in estimates of $b$. There is a subtlety in the choice of parameter $\epsilon$ in Equation 22. If $\epsilon$ is large, the iterative algorithm appears to have a faster convergence rate but at the cost of significant bias. On the other hand, if $\epsilon$ is small, the convergence rate is slow but the solution has small bias. Figure 4 illustrates a concrete example of solving the ARMA model $x(n)-1.9 x(n-1)+1.06 x(n-$ $2)-0.144 x(n-3)=u(n)+0.7 u(n-1)+u(n-2)$ by using our iterative algorithm.

\section{NUMERICAL EXPERIMENTS}

We present simulations for some interesting cases. Theorem 1 asserts that as long as RIP is satisfied, stability assumptions on $H$ hold, and the spikes are well separated, our $\ell_{1}$ minimization algorithm reconstructs the AR process correctly. For general IID Gaussian or Bernoulli matrix ensemble (not Toeplitz), it is well known that [9] $m \geq O(S \log (n / S))$ 

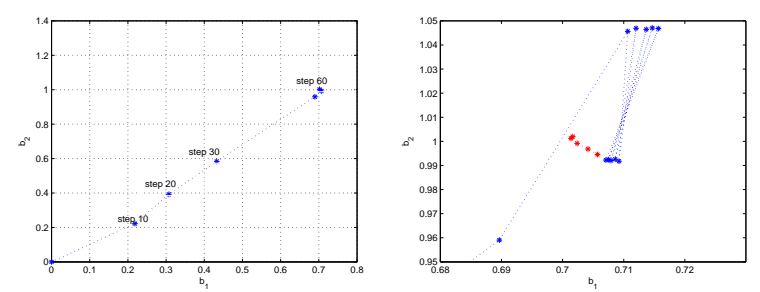

Fig. 4. The iterative algorithm on the model $x(n)-1.9 x(n-1)+$ $1.06 x(n-2)-0.144 x(n-3)=u(n)+0.7 u(n-1)+u(n-2)$ where the correct $b=\left[\begin{array}{ll}0.7 & 1\end{array}\right]$. Left: In trajectory of $\hat{b}$ in each round of iteration; Right: Zoom-in of the final stages of the iterations. Blue $*$ corresponds to the rounds of updates with $\epsilon=3$ while red * corresponds to the rounds of updates with a smaller $\epsilon=0.3$ in the final stage.
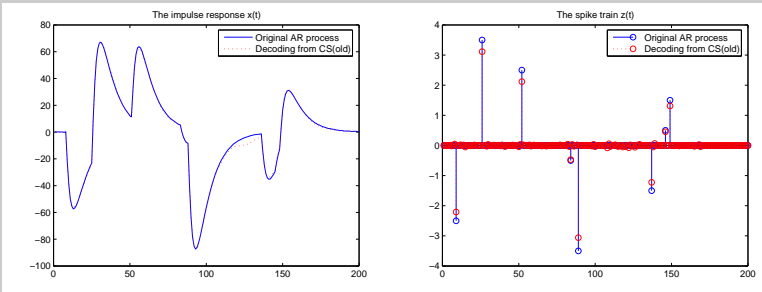

Fig. 5. $\ell_{1}$-minimization algorithm on the model $y=G x+w$ with $G$ an $80 \times 200$ Toeplitz Gaussian matrix ensemble. The filtered process $x(n)$ is obtained by filtering a 8 sparse spike train through a third-order AR process with poles $\alpha_{1}=0.9, \alpha_{2}=0.5$ and $\alpha_{3}=0.2$. The measurements were contaminated with zero mean Gaussian noise with variance 0.1 .

ensures good RIP property. However, for our specific Toeplitz structured sensing matrix (Equation 2), this question (when RIP is satisfied) has not been fully answered. We nevertheless experiment with Toeplitz constructions. First we simulate our algorithm for a third order process. The results are depicted in Figure 5. We see that the reconstruction reproduces both the spike train as well as the filtered process accurately. For the purpose of depiction we added a small amount of noise.

Next we fix the size of sensing matrix $(m=50, n=200)$ and choose the entries of sensing matrix $G$ to be Gaussian or Bernoulli. The order of the AR model is 2 and we let sparsity, $k$, vary from $1--20$. For each fixed $k$, we run our $\ell_{1}$-minimization algorithm 50 times to obtain the average performance. We can see that in this example Toeplitz Bernoulli matrix is preferable to Toeplitz Gaussian matrix. Finally we test impact of AR order influences the performance of the algorithm. We fix the size of the sensing matrix as $80 \times 200$ and also fix the sparsity $k=10$ (i.e., the \# fraction of nonzero components in $u$ is $5 \%$ ). We let order of the AR process vary from 1 to 15 . Again we see that Bernoulli Toeplitz matrix outperforms the Gaussian Toeplitz matrix.

\section{REFERENCES}

[1] E. A. Robinson, "Seismic time-invariant convolutional model," GEOPHYSICS, vol. 50, pp. 2742-2752, December 1985.

[2] W. Gerstner and W. M. Kistler, Spiking Neuron Models Single Neurons, Populations, Plasticity. Cambridge University Press, August 2002.

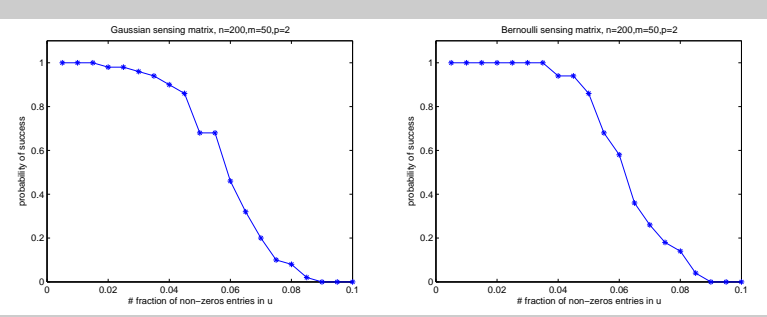

Fig. 6. $\ell_{1}$-minimization algorithm on the model $y=G x$ with $G$ an $50 \times 200$ Toeplitz matrix with independent Gaussian or Bernoulli entries. In this experiment $x(n)$ is a second-order AR process with poles $\alpha_{1}=0.9$ and $\alpha_{2}=0.5$; Left: success rate when $G$ is Gaussian $\mathcal{N}(0,1)$; Right: success rate when $G$ is Bernoulli \pm 1 .

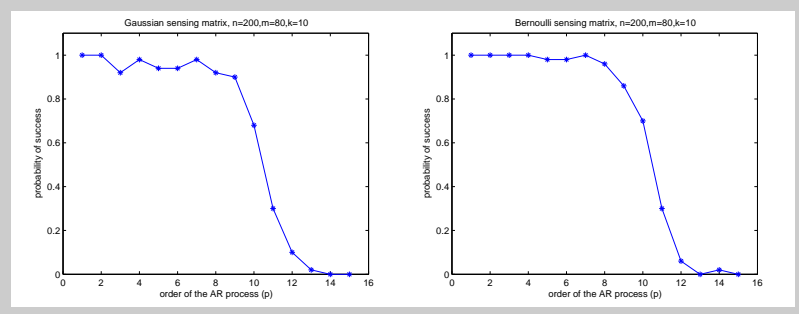

Fig. 7. $\ell_{1}$-minimization algorithm on the model $y=G x$ with $G$ an $80 \times 200$ Toeplitz matrix with independent Gaussian or Bernoulli entries. In this experiment the order of the AR process $x(n)$ is a variable, i.e., $p \in[1,15]$;Left:success rate when $G$ is Gaussian $\mathcal{N}(0,1)$; Right: success rate when $G$ is Bernoulli \pm 1 .

[3] D. L. Snyder, Random point processes. John Wiley and Sons, 1975.

[4] J. Haupt, W. E. Bajwa, G. Raz, and R. Nowak, "Toeplitz compressed sensing matrices with applications to sparse channel estimation," October 2008, preprint.

[5] J. M. Mendel, Optimal Seismic Deconvolution: An Estimation Based Approach. Academic Press, New York, 1983.

[6] D. Donoho, "Compressed sensing," IEEE Transactions on Information Theory, vol. 52, no. 4, pp. 1289-1306, April 2006.

[7] V. Saligrama, "Deterministic designs with deterministic guarantees: Toeplitz compressed sensing matrices, sequence designs and system identification," preprint - 2008.

[8] D. Donoho, "For most large underdetermined systems of linear equations the minimal $\ell^{1}$-norm solution is also the sparsest solution," Communications on Pure and Applied Mathematics, vol. 59, no. 6, pp. 797-829, June 2006.

[9] E. Candès, J. Romberg, and T. Tao, "Stable signal recovery from incomplete and inaccuarte measurements," Communications on Pure and Applied Mathematics, vol. 59, no. 8, pp. 1207-1223, August 2006.

[10] V. Saligrama and M. Zhao, "Compressed blind de-convolution," CoRR, vol. abs/0910.0239, 2009.

[11] C. Andrieu, E. Barat, and A. Doucet, "Bayesian deconvolution of noisy filtered point processes," IEEE Transactions on Signal Processing, vol. 49, no. 1, pp. 134-146, 2001.

[12] E. Candès and Y. Plan, "Near-ideal model selection by $\ell_{1}$ minimization," December 2007, preprint.

[13] B. Alkire and L. Vandenberghe, "Handling nonnegative constraints in spectral estimation," in Proc. IEEE Asilomar Conf. Signals, Systems and Computers, 2000., pp. 202-206.

[14] H. Boche and V. Pohl, "Spectral factorization for polynomial spectral densitiesimpact of dimension," IEEE Transactions on Information Theory, November 2007. 